\title{
mConduct: A Multi-Sensor Interface for the Capture and Analysis of Conducting Gesture
}

\author{
Joanne Armitage, Phoebe Bakanas, Joel Balmer, Paul Halpin, Kyle Hudspeth, Kia Ng \\ ICSRiM - University of Leeds, \\ School of Electronic and Electrical Engineering, School of Music \& School of Computing, \\ Leeds LS2 9JT, UK \\ mconduct@icsrim.org.uk, www.icsrim.org.uk
}

\begin{abstract}
The art of conducting has a long and well-established history, using physical gesture to convey musical intent and expressions. Conducting relies on visual communications to direct the ensemble as a coherent unit. The aim of this project is to capture and analyse the hand gestures of conducting in order to provide real-time, interactive multimodal feedback in a number of application contexts including visualisation. This paper presents the design and development of the interface involving hardware sensors and software analysis modules, and discusses the application of visualisation for conducting. The paper concludes with latest findings, future directions and the impact the research may have outside the realm of gesture communication application.
\end{abstract}

Transdomain. Music via motion. Gesture. Vibrotactile. Visualisation. Conducting. Sensors.

\section{INTRODUCTION}

Conductors direct musical performances through visual gesture. While the performers look to the conductor for tempo, dynamics and unified entrances and exits; audiences look to the conductor for a visual representation of what they hear. The mConduct project is developing a realtime interactive multimedia system that captures and analyses a conductor's gesture to offer multimodal feedback including visualisation, sonification and vibrotactile. The system is designed for several different application scenarios including distributed performance. This paper provides an overview of the system design and development, and focuses on the visualisation feedback aspects.

The overall system can be divided into four modules: (i) input module - first captures data from the conductor; both intricate data such as movement and acceleration, alongside other general gestural data such as pressure; (ii) analysis module - analyses this data to detect features such as beat points, before the; (iii) mapping module maps the analysed data for reconstruction in accordance to the selected mapping strategy; (iv) feedback module - reconstructs data into the appropriate in the chosen application.
Section 2 of the paper presents a background literature survey of the research including an overview of conducting history and technique, conductor tracking systems and visualisation techniques. Section 3 presents the design and development of the system. Section 4 discusses evaluations and validations of the system, and the final section concludes the paper with latest findings, future developments and the impact this research may have outside the realm of conducting and gesture communication.

\section{BACKGROUND}

This section presents a brief historical progression of conducting practice in relation to the development of musical form in order to contextualise the development of mConduct.

\subsection{Conducting}

\subsubsection{History of conducting}

In the context of this project, we look at the role of a conductor, using arm and hand gesture to coordinate a group of players to perform with precise and delicate synchronisation and timing. This gestural communication is required to control a range of musical parameters; for example, indicating entries, setting tempo, synchronising the 
various instruments and parts and shaping the ensemble's sound.

The role of the conductor evolved slowly through a variety of practices, influenced by political, economic and technological changes (Bowen 2003). The earliest form of conducting, cheironomy, can be traced back to early Egyptian performances. Before melody was notated in a written score, cheironomy hand signs indicated melodic shape of phrases (Ranhofer 2004). Cheironomy was widespread in the ancient world and it endured into medieval times, being used to direct singers of Gregorian chant (Haïk-Vantoura 2011). However, during the Middle Ages, music became more complex. The melodic line expanded to have more pitches sung upon a single syllable as well as more pitches in the contour of the melody. The potential pitches within the octave also expanded allowing ensembles to have looser tonality. In addition to the development of polyphony, music also became more rhythmically complex. Cheironomy could not facilitate this development and lost its effectiveness. The complexity of polyphonic music compelled the development of staffed musical notation (Haïk-Vantoura 2011, Medieval and Renaissance Music 2011).

As music became more complex, a staff was used to indicate the beat instead of pitch, thus acting as an early form of a baton (Conducting 2011). One of the earliest reports of a conductor using a staff to keep an ensemble together is from 709 BC. (Bowen 2003) Pherekydes of Patrae "giver of Rhythm" waved a golden staff up and down to keep an ensemble of eight hundred performers in time and together. However, it was in Medieval choirs that role of the staff as a conductor's tool gained prominence. Its use spread to other ensembles in the 16th and 17th century. It is reported that JeanBaptiste Lully (1632 -1687), the Maitre de musique for Louis XIV, used a very large stick that he banged on the floor to keep time. During the 18th century developments in technology enabled lower production costs for both printing and instrument manufacturing. Political and economic changes ended the wealth of many patrons and disbanded their orchestras, thus creating a new middle class market for musicians. Composers now composed for a new and unstudied public and no longer had control of how their works were performed. Thus, the position of a conductor as a director of a performance surfaced simultaneously with the rise of the independent musicians. In the 18th century the form of the conducting device evolved from the staff to a rolled up piece of paper and then the baton in the 19th century (Bowen 2003).

During the first half of the 19th century conductors experimented with both their technique and role in an ensemble. Aspects such as audible vs silent beats, different forms of divided leadership, as well as where to stand, which way to face and what to hold were explored. There were many discourses on how to bring order as larger ensembles struggled to play increasingly complex music (Bowen 2003). By the mid 19th century, it became common practice to have a dedicated conductor, an individual who did not play in the ensemble.

In the 19th century Wagner developed a theory that shaped the role of conductors today (Marrin 1996). He believed that conductors should not only keep time but also impose their own interpretation of the piece. Modern conducting is built upon the traditional techniques founded by Wagner and other early conductors. However, conducting is still an evolving art form. Not only does modern music necessitate the use of new conducting techniques but also technology is opening doors for new types of music and interpretations of conducting.

\subsubsection{Conducting technique}

The rise of rhythmically complicated polyphonic music made it necessary to have a conductor to coordinate different parts of an ensemble with a visible pulse. The 16th century had many written discourses argue over the best way to mark the tactus. In 1701 lexicographer, Thomas Janowka, described tactus for an ordinary measure as a right-hand movement of down, left, right, up. This pattern became the standard and is the basis of modern conducting methods.

Today, the beat of the music is normally indicated with the conductor's right hand using a baton. The baton traces a shape in the air for every measure indicating each beat with a sudden change in baton direction. The shape is dependent on the time signature. A strong down movement indicates the first beat of the bar and a distinct upward movement indicates the last beat of the bar (Rudolf \& Stern 1994).

While some conductors may use both hands to indicate or highlight the beat, with the left hand mirroring the right, the second hand is normally used for cueing the entrances of individual players or sections and aid indications of dynamics, phrasing, expression, and other elements (Rudolf \& Stern 1994).

\subsection{Conductor tracking systems}

A variety of conducting gesture analysis and performance systems have been developed. The first documented mechanisation of the conducting baton was during the 1830s in Brussels. The system relayed the conductor's tempo to an offstage chorus through an electromechanical 
device, similar to a piano key. This would complete an electrical circuit and turn on a light when pressed. Hector Berlioz documented the use of this device in his essay, 'On Conducting,' published in 1843.

There have been many attempts in conductor tracking systems. The most common objective for these systems is conducting electronic instruments or a virtual orchestra. The term 'virtual orchestra' was first introduced into the musical lexicon in the early 1990s by Bianchi \& Smith (2011). Bianchi and Smith developed an interactive computer music system that was used in the Kentucky Opera's 1995 production of Hansel and Gretel. This marked one of the first uses of technology by a major performing arts organisation.

Another example of an early virtual orchestra is the system created by Morita, Hashimoto and Ohteru (1991). Their electronic orchestra responded to a conductor's gestures. Movements were tracked through a Charge-Coupled Device (CCD) camera and sensor glove. Morita et al. categorise the tracked conducting information into two main functions:

i) Basic that includes notes, pitch, frequency, duration.

ii) Musical performance expression, such as ritardando, sostenuto, dolce.

The basic information is quantifiable and necessary when performing a piece. The expressive information is subjective and creates the artistic essence of the performance (Morita, Hashimoto \& Ohteru 1991). Ascertaining beat points to indicate tempo is a minimum requirement for this system. Expanding upon this to measure gestural expression is fundamental in creating an authentic reconstruction.

In 1996, the 'Digital Baton' (Marrin 1997, Marrin \& Paradiso 1997) was designed as a multipurpose device to control electronic music through traditional conducting parameters such as tempo, dynamic and duration alongside individual notes and details of particular sounds. Gestures are tracked using accelerometers, infrared light emitting diodes (LEDs) and piezo-resistive strips.

While many conducting gesture analysis systems focus on controlling a virtual orchestra, others are motivated by conductor analysis research. Nakra (2000) presents the 'Conductor's Jacket'. The 'Conductor's Jacket' is a physiological monitoring system built into the clothing of a conductor; it is designed to study conductors' techniques in their working environments.
Other systems aim at conducting pedagogy. Examples include Peng and Gerhard (2009) and Bradshaw and Ng's (2008, 2009) conducting analysis systems. These two projects used a Wiibased tracking system to capture the conducting gesture.

Many other conducting tracking systems have been developed including: Lee, Narka \& Borchers (2004); Borchers et al. (2004); Katayose and Okudaira (2004); Bruegge et al. (2007); Nakra et al. (2009); Baba, Hashida \& Katayose (2010).

\subsection{Mapping and visualisations}

Data mapping involves the translation of data across different domains. In this system mapping is implemented to convert gestural conducting data using several feedback technologies including visualisation. Through effective mappings, visualisation can enhance aspects of the data that are not apparent in its raw form. The techniques have been applied in a wide range of application contexts including technology-enhanced learning. For example, Oliver \& Aczel (2002) and Ng (2011) reported accelerated learning using visualisation.

$\mathrm{Ng}$ et al. (2007) and $\mathrm{Ng}$ (2011) discusses the i-Maestro 3D Augmented Mirror system which increases awareness of bowing gesture (for string instruments) and body posture using real-time visualisation and sonification. MacRitchie, Buck and Bailey (2009) visualise musical structure through motion capture of a pianist's performance gestures. This visualisation confirmed a relationship between upper body movements of a pianist and composition structure. Robertson, Stark and Plumbley (2011) designed a beat tracking algorithm for real-time beat visualisation.

Visualisation of sound is commonplace in both performance and personal listening environments. Generative, real-time visualisations of audio are often integrated into media players. In a performance environment, audiovisual composition explores the relationship between sonic and visual material and generally combines electronic or electroacoustic audio with video footage or computer graphics (Miyama 2011).

Conducting gesture is an effective way of deriving visualisations that relate to sound due to it encompassing all aspects relating to the performance. 


\section{DESIGN AND DEVELOPMENT}

\subsection{Requirement}

In this section, several design requirement considerations are discussed on the measurement, analysis and reconstruction of conducting gesture.

To realise the aim of the project, the system needs to be able to capture the delicate motions of the conductor with sufficient accuracy and precision required for the analysis for the visualisation. The sensors measuring the conductor's motion must be sensitive enough to capture sufficient detail in the gesture. A non-intrusive sensing hardware is required so that the baton is not altered too significantly in terms of size and weight.

The system requires algorithms that are capable of analysing and detecting changes in motion, particularly at the turning point of the gesture where the conductor is indicating a beat.

To integrate the hardware and software components of the design, data communication is one of the key aspects. A minimum baud rate of 57600 bit per second is required to allow a reliable connection up to 200 frame per second, assuming each frame include up to nine 32 bits floating-point data (such as 3D accelerometer data etc).

The system also requires a set of different visual mapping strategies in order to translate the detected gesture features to visual parameters.

The project is particularly interested in visualisation strategies that emphasises and highlights differences in conducting gesture to be able to show different expressions and interpretations.

\subsection{Development}

The characteristics of the conductor's gesture are determined using multisensory data fusion. In order to capture the necessary data, the system uses an inertial measurement unit (IMU), consisting of an integrated accelerometer, gyroscope, and magnetometer, attached to the base of a conductor's baton. A separate design has been implemented for users who conduct without a baton. The device was designed to be as lightweight and non intrusive as possible. The accelerometer was programmed to have a sensitivity of $\pm 4 \mathrm{~g}$, this gives a good balance between sensitivity and maximum force.

The IMU unit measures 3D vectors depending on the orientation of the sensor board. In order find the global movement, we compute quaternions $\left(q_{1}, q_{2}\right.$, $q_{3}, q_{4}$ ) using the accelerometer and gyroscope data
(Diebel 2006). The effect of gravity on the accelerometer is calculated ( $g x, g y, g z)$ using the quaternions:

$$
\begin{aligned}
& g x=2\left(q_{2} q_{4}-q_{1} q_{3}\right) \\
& g y=2\left(q_{1} q_{2}+q_{3} q_{4}\right) \\
& g z=q_{1}^{2}-q_{2}^{2}-q_{3}^{2}+q_{4}^{2}
\end{aligned}
$$

These gravity values are then subtracted from the raw accelerometer data to give gravitycompensated accelerometer values.

From the quaternions, a rotation matrix is computed in order to transform the accelerometer data onto a global coordinate system, regardless of device orientation. This is achieved by deriving Euler angle values for the yaw $(\psi)$, pitch $(\theta)$, and roll $(\varphi)$ of the device from the quaternions (Madgwick 2010):

$$
\begin{aligned}
& \psi=\operatorname{atan} 2\left(2 q_{2} q_{3}-2 q_{1} q_{4}, 2 q_{1}^{2}+2 q_{2}^{2}-1\right) \\
& \theta=-\sin ^{-1}\left(2 q_{2} q_{4}+2 q_{1} q_{3}\right) \\
& \Phi=\operatorname{atan} 2\left(2 q_{3} q_{4}-2 q_{1} q_{2}, 2 q_{1}^{2}+2 q_{4}^{2}-1\right)
\end{aligned}
$$

These Euler angles are then used in a rotation matrix to calculate acceleration values relative to the world coordinate system (LaValle 2006).

This is all performed on board the Arduino system (http://www.arduino.cc/). The accelerometer data is then used to determine the strength and intensity of a conductor's gestures, whilst gyroscope data is used to determine the directionality of these motions. These two data streams together provide an in-depth representation of the baton's movement.

After the sensor data has been processed and analysed, the information is broadcast wirelessly, using the ZigBee wireless protocol, to computers for use in the visualisation and sonification systems, as well as the actuator unit that physically translates the motion to vibration. The system adopts the 'Music via Motion' (MvM) framework design that facilitates the trans-domain mapping of movement to another multimedia domain $(\mathrm{Ng}$ 2004). The modular architecture of the systems using MvM has influenced the design of this system particularly in respect to its potential for multiple applications and multimodal reconstructions.

The system design enables the stream of gestural information to be broadcast and received by multiple actuator units and computers in order to provide distributed processing and multimodal 
feedback. The visualisation software receives a real-time data stream through the serial port. Data is then analysed and mapped to visual parameters including three dimensional shape, size and colour. The gyroscope data informs shape boundaries and size. 3D acceleration data is mapped to red, green and blue pixel intensity values. This mapping strategy visualises repetition patterns of the acceleration in the gesture through clusters of colour. The overall intention of the visualisation is to create a three-dimensional sculpture of the user's overall gesture. This encompasses musical parameters such as structure, expression, tempo and time signature.

The visualisation software contains user-definable controls that allow fine-tuning and custom display modes for greater freedom in performance. Camera view settings in software allow the user adjustable zoom and other 3D controls. Snapshots of the shape can be taken throughout the performance for comparative analysis of specific sections of a performance, see Figure 2 for an example output.

The shape of the 3D graphical sculpture creates a clear visual distinction between the different time signatures. The number of distinct 'loops' visualised is congruent with the number of beats per measure. The colour mapping strategy of the acceleration data identifies clusters of colour at the same position on different measures. The distribution of colour intensity suggests the user's gestural accuracy and consistency.

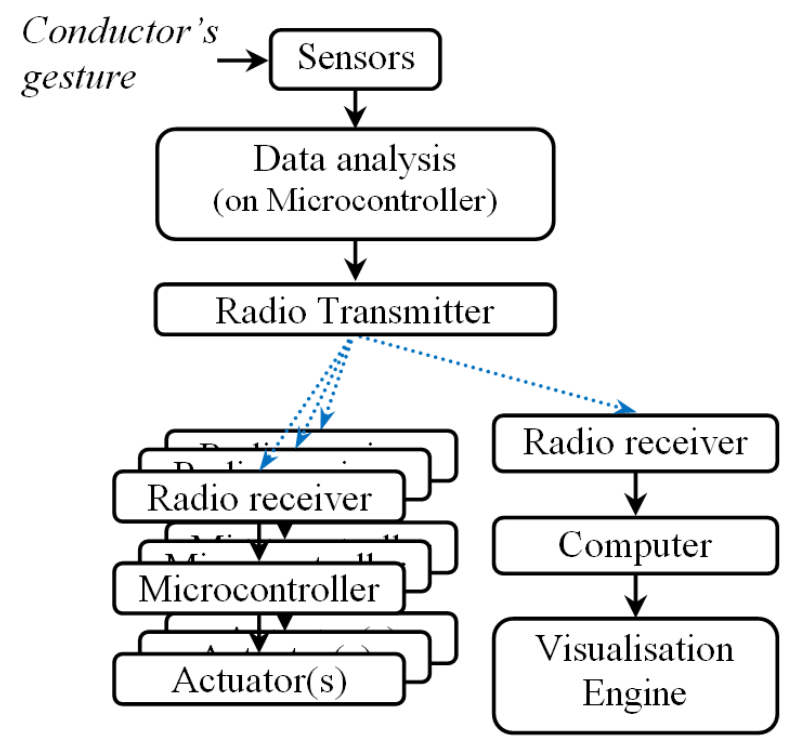

Figure 1: Overall system architecture
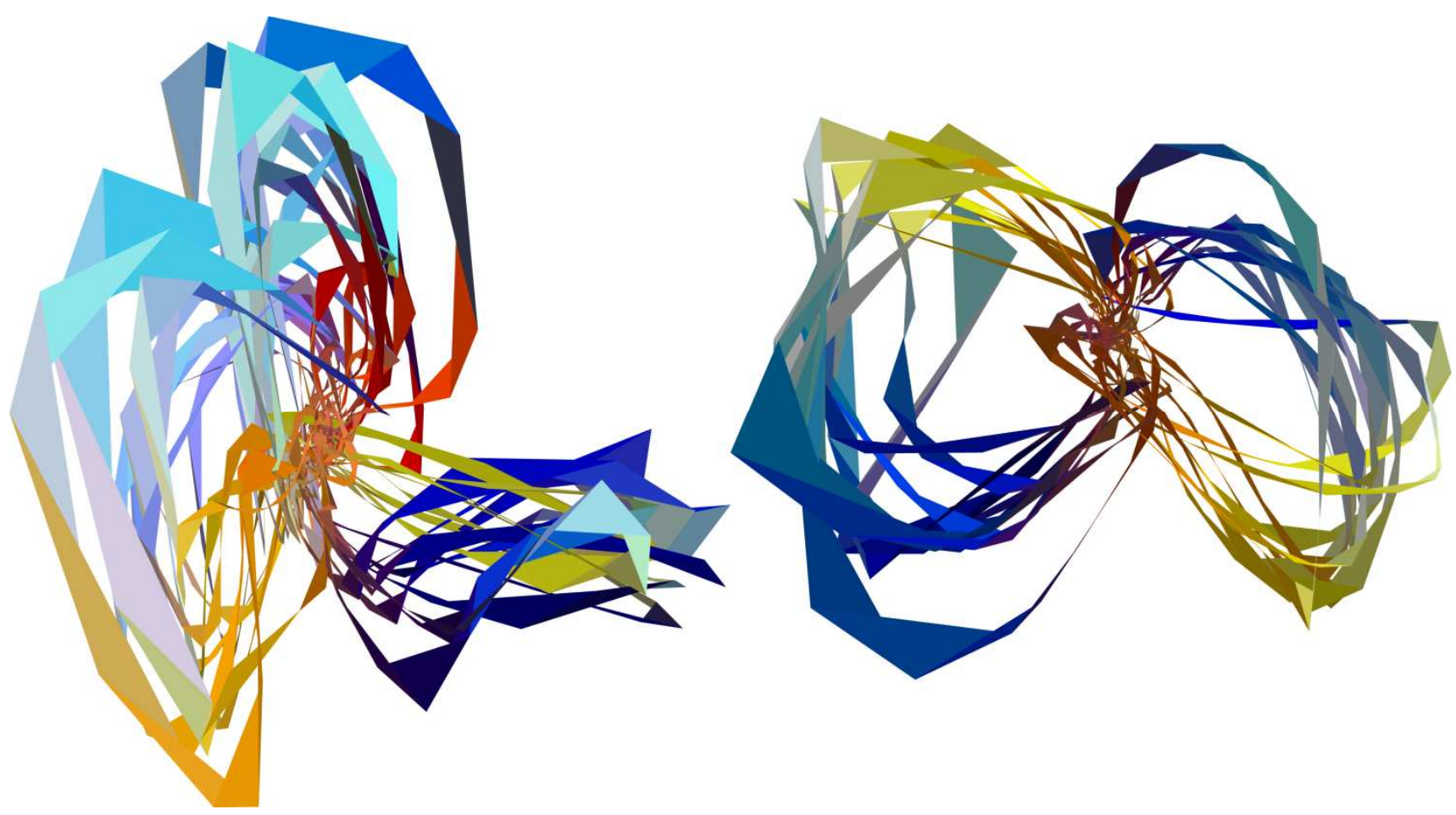

Figure 2: Left shows example visualisations for a sequence of gesture in 3/4 time and right in 2/4 time 


\section{VALIDATION}

To ascertain the system's performance and establish the systems capabilities tests and validation procedures are applied to assess the speed, jitter and accuracy.

Trial runs of evaluations were performed and refined for a user group to ensure they were accurate and meaningful. The subjects were given a questionnaire, which outlined questions relating to system practicality and user experience. Initial evaluations have verified the system as sufficient for the purposes specified above.

The set of visualisation mapping have been designed to convey features of the data set and communicate the motions in a recognisable and meaningful form. The visualisation module presents conducting gesture through geometric shapes, colours and other visual features.

Initial tests have found that the visualisation can provide insightful understanding of the gesture by comparing the sculpture-like graphical output. This is currently being trailed as an aid within a technology-enhanced learning system in Leeds. We are also working with a composer who is writing a piece that combines the visualisation element of the system into a performance.

\section{CONCLUSION}

This paper proposed the mConduct system to capture and analyse the hand gestures of conducting in order to provide real-time, interactive multimodal feedback with particular focus on visualisation.

The paper reviewed the evolution of conducting and the baton; discussed the design and development of the tool involving multiple hardware sensors and software analysis modules; examined the parameters for the visualisation software; and discussed evaluations and validations and next steps in the development.

With initial evaluations, we believe the mConduct system can increase conductor communication, aid pedagogical purposes and provide a means for gesture comparative analysis.

Anglin (2004), Arcavi (2003), Lewalter (2002), Winn (1982) and Leavitt (2012) proposed that people remember information when it is represented and learn both visually and audibly. The use of visuals helps build mental models by directing attention to important information and organizing data in a meaningful way. Visuals and corresponding auditory information are integrated into one comprehensive mental model. The mental model can be a powerful tool in pedagogical context for the interpretation and understanding of a performance. Conducting gesture visualisations were found to have a similar impact when combined with live audio. Together they provide an enhanced mental model of the performance elements including expression, tempo, time signature and mood.

Visualisation of conductors' gesture expands live performance by engaging the audience on two levels. The visuals created by mConduct can be used to summarize a conductor's performance. Both conducting patterns and deviations from the normal conducting movements are represented. Students can use the mConduct system to visualise their own conducting patterns and evaluate their consistency and variation of gesture as well as compare their motions to others.

The visualisation of gestures can also be used for comparative analysis. The images formed from the visualisation can be stored and then compared. This can be used for technology-enhanced learning: First, a user can evaluate their consistency by comparing visuals from a series of performances of the same piece. Secondly, a user can track their development by looking at visuals of their conducting over time. Third, users can compare the visual of their performance to others' in order to study differences in techniques and interpretations.

The value of mConduct goes beyond gesture communication applications. The system will benefit cultural heritage through performance preservation. The collected data, analysis and visualisations can be stored for future generations. Future musicians can retrieve the visualisation data and discover how previous conductors shaped a piece.

\section{REFERENCES}

Conducting

(2011)

Conducting. http://www.newworldencyclopedia.org/entry/Condu cting_\%28music\%29 (12 Dec 2011).

Medieval and Renaissance Music (2011) Medieval and Renaissance Music. http://www.essentialhumanities.net/index.php (20 Jan 2012).

Anglin, G.J, Vaez, H. and Cunningham, K.L. (2004) Visual Representations And Learning: The Role Of Static And Animated Graphics. Handbook of research on educational communications and technology, pp. 865-916. 
Arcavi, A. (2003) The role of visual representations in the learning of mathematics. Educational Studies in Mathematics, 52(3), pp. 215-241.

Baba, T., Hashida, M. and Katayose, H. (2010) VirtualPhilharmony: A Conducting System with Heuristics of Conducting an Orchestra. NIME, 2010, pp. 263-270.

Bianchi, F. and Smith, D.B. (2011) Virtual Orchestra.

http://www.virtualorchestra.com/index.html (Dec. 2011).

Borchers, J., Lee, E., Samminger, W. and Mühlhäuser, M. (2004) Personal orchestra: a realtime audio/video system for interactive conducting. Multimedia Systems, vol. 9, pp. 458 - 465.

Bowen, J. A. (2003) The Rise of Conducting. The Cambridge Companion to Conducting. Cambridge University Press.

Bradshaw, D. and Ng, K. (2009) Motion Capture, Analysis and Feedback to Support Learning Conducting. International Computer Music Conference, McGill University, Montreal, Canada, August 2009,16 - 21.

Bradshaw, D. and Ng, K. C. (2008) Analyzing a conductor's gestures with the Wiimote. EVA, 2008, pp. 35-42.

Bradshaw, D. J. (2009) Interactive Multimodal Feedback of Conducting Gesture. MA Thesis, University of Leeds, 2009.

Bruegge B., Teschner, C., Lachenmaier, P., Fenzl, E., Schmidt, D. and Bierbaum S. (2007) Pinocchio: conducting a virtual symphony orchestra. International Conference On Advances In Computer Entertainment Technology, 2007.

Diebel, J. (2006) Representing Attitude: Euler Angles, Unit Quaternions, and Rotation Vectors. Technical report, Stanford University.

Haïk-Vantoura, S. (2011) Chironomy in the ancient world.

http://www.rakkav.com/biblemusic/pages/chironom y.htm (28 Jan. 2012).

Hogan, K. and Stubbs, R. (2003) Can't get Through 8 Barriers to Communication. Pelican Publishing Company, Grenta, LA.

Katayose, H. and Okudaira, K. (2004) iFP A Music Interface Using an Expressive Performance Template. Entertainment Computing, vol. 3166, pp. 529-540.

Lavalle, S. (2006) Planning Algorithms. Cambridge University Press.

Leavitt, M. (2012) Learning From Visuals. How Well-Designed and Well-Used Visuals Can Help Students Learn. Wiley Visualizing.
Lewalter, D. (2002) Cognitive strategies for learning from static and dynamic visuals. Learning and Instruction, 13(2), pp. 177-189.

Lee E., Nakra, T.M. and Borchers, J. (2004) You're the conductor: a realistic interactive conducting system for children. New Interfaces For Musical Expression, Hamamatsu, Japan, 2004, p. 6. M. Rudolf and M. Stern, The grammar of conducting: A comprehensive guide to baton technique and interpretation, 3rd ed.: Schirmer Books, 1994.

MacRitchie, J., Buck, B. and Bailey, N.J. (2009) Visualising Musical Structure Through Performance Gesture. 10th International Society for Music Information Retrieval Conference, 2009.

Madgwick, S. (2010) An Efficient Orientation Filter for Inertial and Inertial/Magnetic Sensor Arrays. Technical report,

Department of Mechanical Engineering, University of Bristol.

Marrin, T. (1996) Toward an Understanding of Musical Gesture: Mapping Expressive Intention with the Digital Baton. Master's thesis, MIT Media Lab, Boston.

Marrin, T. (1997) Possibilities for the Digital Baton as a General Purpose Gestural Interface. CHI, 1997, pp. 311-312.

Marrin, T. and Paradiso, J. (1997) The Digital Baton: a Versatile Performance Instrument. International Computer Music Conference, Thessaloniki, Greece, 1997.

Miyama, C. (2011). Quicksilver. International Computer Music Conference.

Morita, H., Hashimoto, S. and Ohteru S. (1991) A Computer Music System that Follows a Human Conductor. Computer, vol. 24, pp. 44-53.

Nakra, T.M. (2000) Inside the Conductor's Jacket: Analysis, Interpretation and Musical Synthesis of Expressive Gesture. Massachusettes Institute of Technology, Boston, MA.

Nakra, T.M., Ivanov, Y., Smaragdis, P. and Ault, C. (2009) The USB Virtual Maestro: an Interactive Conducting System. NIME, 2009.

$\mathrm{Ng}, \mathrm{K}$. C. (2004) Music via motion: Transdomain mapping of motion and sound for interactive performances. IEEE, Mar. 2004, pp. 645-655.

$\mathrm{Ng}, \quad \mathrm{K}$., Interactive Multimedia for Technologyenhanced Learning with Multimodal Feedback, Musical Robots and Interactive Multimodal Systems, Springer Tracts in Advanced Robotics, Springer, isbn 3642222900, 2011.

Ng, K., Weyde, T., Larkin, O., Neubarth, K., Koerselman, T. and Ong, B. (2007) 3D Augmented Mirror: A Multimodal Interface for String Instrument 
Learning and Teaching with Gesture Support. 9th international conference on Multimodal interfaces, 2007, pp 339 - 345. ACM.

Oliver, M. and Aczel, J. (2002) Theoretical Models of the Role of Visualisation in Learning in Formal Reasoning. Journal of Interactive Media in Education.

Peng, L. and Gerhard D. (2009) A wii-based gestural interface for computer-based conducting systems. New Interfaces For Musical Expression.

Randhofer, R. (2004) By the Rivers of Babylon: Echoes of the Babylonian Past in the Musical
Heritage of the Iraqi Jewish Diaspora. Ethnomusicology Forum, vol. 13, pp. 21-45.

Robertson, A., Stark, A. M. and Plumbley, M. D. (2011) Real-time Visual Beat Tracking using a Comb Filter Matrix. International Computer Music Conference, 2011, pp. 617-620.

Winn, W. (1982) Visualization in learning and instruction: a cognitive approach. Educational Technology Research and Development, 30(1). pp 3-25. 